\title{
Strong practical stability and stabilization of differential linear repetitive processes
}

\author{
Pawel Dabkowski ${ }^{\mathrm{a}, *}$, Krzysztof Galkowski $^{\mathrm{a}}$, Eric Rogers ${ }^{\mathrm{b}}$, Olivier Bachelier ${ }^{\mathrm{c}}$ \\ a Institute of Physics, Nicolaus Copernicus University, Torun, Poland \\ ${ }^{\mathrm{b}}$ School of Electronics and Computer Science, University of Southampton, Southampton, SO17 1BJ, UK \\ ${ }^{c}$ LAII, ESIP, University of Poitiers, 40 Avenue du Recteur Pineau 86022 Poitiers Cedex, France
}

\section{A R T I C L E I N F O}

\section{Article history:}

Received 15 March 2010

Received in revised form 2 August 2010

Accepted 6 August 2010

Available online 15 September 2010

\section{Keywords:}

2D systems dynamics

Stability

Control law design

\begin{abstract}
A B S T R A C T
Differential linear repetitive processes evolve over a subset of the upper-right quadrant of the 2D plane where the unique feature is a series of sweeps or passes through a set of dynamics governed by the solution of a linear matrix differential equation over a finite duration $t \in[0, \alpha]$ where $\alpha$ is termed the pass length or duration. On each pass an output, termed the pass profile, is produced, which acts as a forcing function on, and hence contributes to, the dynamics of the next pass profile. The result can be oscillations in the pass-to-pass direction that cannot be controlled by direct application of standard, or 1D linear systems theory. The existing stability theory demands a bounded-input bounded-output property uniformly, which in the case of the along-the-pass dynamics means for $t \in[0, \infty]$ and for $(k, t) \in[0, \infty] \times[0, \infty] \supset[0, \infty] \times[0, \alpha]$ where the integer $k \geq 0$ denotes the pass number or index. The pass length is always finite, however, and hence this stability theory could well be too strong in many cases and, in particular, impose very strong conditions in terms of control law design. This paper develops an alternative in such cases by relaxing the requirement for the bounded-input bounded-output property to hold when $k \rightarrow \infty$ and $t \rightarrow \infty$ simultaneously, provides an explanation of the implications of this in the frequency domain, and then develops control law design algorithms.
\end{abstract}

(c) 2010 Elsevier B.V. All rights reserved.

\section{Introduction}

The unique characteristic of a repetitive process [1] is a series of sweeps, termed passes, through a set of dynamics defined over a fixed finite duration known as the pass length. On each pass an output, termed the pass profile, is produced which acts as a forcing function on, and hence contributes to, the dynamics of the next pass profile. This, in turn, leads to the unique control problem where the output sequence of pass profiles generated can contain oscillations that increase in amplitude in the pass-to-pass direction.

To introduce a formal definition, let $\alpha<+\infty$ denote the pass length. Then in a repetitive process the pass profile $y_{k}(t), 0 \leq t \leq$ $\alpha$, generated on pass $k$ acts as a forcing function on, and hence contributes to, the dynamics of the next pass profile $y_{k+1}(t), 0 \leq$ $t \leq \alpha, k \geq 0$.

Physical examples of these processes include long-wall coal cutting and metal rolling operations [1]. Also applications have arisen where adopting a repetitive process setting for analysis

\footnotetext{
* Corresponding author.

E-mail addresses: p.dabkowski@fizyka.umk.pl (P. Dabkowski), etar@ecs.soton.ac.uk (E. Rogers), Olivier.Bachelier@univ-poitiers.fr (O. Bachelier).
}

has distinct advantages over alternatives. Examples of such algorithmic applications include classes of iterative learning control schemes, where one possible source for the literature in this area is the survey papers [2,3], and iterative algorithms for solving nonlinear dynamic optimal stabilization problems based on the maximum principle [4]. In this latter case the use of the repetitive process setting provides the basis for the development of highly reliable and efficient iterative solution algorithms. More recently, iterative learning control algorithms designed using linear repetitive process stability theory have been experimentally validated [5] on a gantry robot whose task is to place a sequence of objects onto a moving conveyor under synchronization, confirming that this approach can remove performance related problems with other designs.

Recognizing the unique control problem, the stability theory for linear repetitive processes is of the bounded-input boundedoutput (BIBO) form, that is a bounded initial pass profile is required to produce a bounded sequences of pass profiles, where the bounded property is defined in terms of the norm on the underlying Banach space. Asymptotic stability in the pass-to-pass direction demands this BIBO stability property over the finite and fixed pass length, that is, over $(k, t) \in[0, \infty] \times[0, \alpha]$, and if this property holds then the sequence of pass profiles generated converges strongly to the limit profile that in some cases, including 
the processes considered here, is described by a standard, or 1D, linear system. The finite pass length does, however, mean that this limit profile can have unacceptable along-the-pass dynamics, including the case when it is unstable.

The most obvious way to exclude this last possibility is to demand the BIBO stability property uniformly with respect to the pass length, that is, over $(k, t) \in[0, \infty] \times[0, \infty]$, and this is termed stability along-the-pass. Moreover, for the processes considered here, the abstract model based stability conditions can be transformed into conditions that can be checked by 1D linear systems stability tests. In the case of asymptotic stability in the pass-to-pass direction, the condition reduces to computing the eigenvalues of a matrix and for stability along-the-pass the extra conditions are an eigenvalue computation for a matrix plus, in the single-input single-output (SISO) case for simplicity, a condition expressed in terms of the frequency content of the initial pass profile. In control law design terms this is a very stringent condition to meet and in this paper strong practical stability is developed as an alternative where in the SISO case the frequency attention is no longer required over the complete frequency range. The approach used is to relax the requirement for the BIBO property to hold when $k \rightarrow \infty$ and $t \rightarrow \infty$ simultaneously, where this combination of $k$ and $t$ cannot arise in a physical application. The analysis is then extended to control law design where the calculations can be undertaken using Linear Matrix Inequalities (LMIs).

Throughout this paper, the null and identity matrices with the required dimensions are denoted by 0 and $I$, respectively. Moreover, $M>0(<0)$ denotes a real symmetric positive (negative) definite matrix.

\section{Background}

The state-space model of a differential linear repetitive process [1] has the following form over $0 \leq t \leq \alpha, k \geq 0$,

$\dot{x}_{k+1}(t)=A x_{k+1}(t)+B u_{k+1}(t)+B_{0} y_{k}(t)$,

$y_{k+1}(t)=C x_{k+1}(t)+D u_{k+1}(t)+D_{0} y_{k}(t)$.

Here on pass $k, x_{k}(t)$ is the $n \times 1$ state vector, $y_{k}(t)$ is the $m \times 1$ pass profile vector and $u_{k}(t)$ is the $l \times 1$ vector of control inputs, and $\alpha<\infty$ is termed the pass length. To complete the process description, it is necessary to specify the boundary conditions, that is, the state initial vector on each pass and the initial pass profile. For the purposes of this paper, no loss of generality occurs from assuming state initial vectors of the form $x_{k+1}(0)=d_{k+1}, k \geq 0$, where the $n \times 1$ vector $d_{k+1}$ has known constant entries, and initial pass profile $y_{0}(t)=f(t)$, where $f(t)$ is an $m \times 1$ vector whose entries are known functions of $t$ over $0 \leq t \leq \alpha$.

The stability theory [1] for linear repetitive processes is based on an abstract model in a Banach space setting which includes a wide range of such processes as special cases, including those described by (1). Let $E_{\alpha}$ be a Banach space, $W_{\alpha}$ a linear subspace of $E_{\alpha}$, and $L_{\alpha}$ a bounded linear operator mapping $E_{\alpha}$ into itself. Then the dynamics of a linear repetitive process of constant pass length $\alpha>0$ are described by linear recursion relations of the form

$y_{k+1}=L_{\alpha} y_{k}+b_{k+1}, \quad k \geq 0$,

where $y_{k}$ is the pass profile on pass $k, L_{\alpha}$ is a bounded linear operator mapping $E_{\alpha}$ into itself, and $b_{k+1} \in W_{\alpha}, k \geq 0$. The term $L_{\alpha} y_{k}$ represents the contribution of pass $k$ to pass $\bar{k}+1$ and $b_{k+1}$ represents initial conditions, disturbances and control input effects.

In the case of processes described by (1)

$$
\begin{aligned}
y_{k+1}(t)= & C \int_{0}^{t} \mathrm{e}^{A(t-\tau)}\left(B_{0} y_{k}(\tau)+B u_{k+1}(\tau)\right) \mathrm{d} \tau+C \mathrm{e}^{A t} d_{k+1} \\
& +D_{0} y_{k}(t)+D u_{k+1}(t), \quad 0 \leq t \leq \alpha .
\end{aligned}
$$

Suppose also that $E_{\alpha}$ is the space of bounded continuous mappings of the interval $0 \leq t \leq \alpha$ into the vector space of complex vectors $\mathbb{C}^{m}$ with norm $\|y\|=\sup _{0 \leq t \leq \alpha}\|y(t)\|^{\prime}$, where $\|\cdot\|^{\prime}$ is any convenient norm in $\mathbb{C}^{m}$. Then

$$
\left(L_{\alpha}\right) y(t)=C \int_{0}^{t} \mathrm{e}^{A(t-\tau)} B_{0} y_{k}(\tau) \mathrm{d} \tau+D_{0} y_{k}(t), \quad 0 \leq t \leq \alpha,
$$

and

$b_{k+1}=C \mathrm{e}^{A t} d_{k+1}+C \int_{0}^{t} \mathrm{e}^{A(t-\tau)} B u_{k+1}(\tau) \mathrm{d} \tau+D u_{k+1}(t)$.

Given the unique control problem, the natural approach to a definition of stability for these processes is to ask that given any initial profile $y_{0}$ and any disturbance $b_{k+1}$ that converges strongly to $b_{\infty}$ as $k \rightarrow \infty$, the sequence of pass profiles converges to $y_{\infty}$ as $k \rightarrow \infty$. This is termed asymptotic stability in the pass-to-pass direction of (2) and it can be shown [1] to be equivalent to the existence of real scalars $M_{\alpha}>0$ and $\lambda_{\alpha} \in(0,1)$ such that

$\left\|L_{\alpha}^{k}\right\| \leq M_{\alpha} \lambda_{\alpha}^{k}, \quad k \geq 0$

Equivalently asymptotic stability in the pass-to-pass direction holds for (2) if, and only if, the spectral radius of $L_{\alpha}$, denoted by $r\left(L_{\alpha}\right)$, satisfies $r\left(L_{\alpha}\right)<1$. Moreover, if this condition holds $y_{\infty}$, termed the limit profile, is given by

$y_{\infty}=\left(I-L_{\alpha}\right)^{-1} b_{\infty}$,

where $I$ denotes the identity operator in $E_{\alpha}$ and $b_{\infty}$ the strong limit of $\left\{b_{k}\right\}_{k \geq 1}$ as $k \rightarrow \infty$.

For processes described by (1) it has been shown [1] that asymptotic stability in the pass-to-pass direction holds if, and only if, $r\left(D_{0}\right)<1$. Also if this condition holds and the input sequence applied $\left\{u_{k+1}\right\}_{k}$ converges strongly as $k \rightarrow \infty$ to $u_{\infty}$, the resulting limit profile is described by

$$
\begin{aligned}
\dot{x}_{\infty}(t)= & \left(A+B_{0}\left(I-D_{0}\right)^{-1} C\right) x_{\infty}(t) \\
& +\left(B+B_{0}\left(I-D_{0}\right)^{-1} D\right) u_{\infty}(t), \\
y_{\infty}(t)= & \left(I-D_{0}\right)^{-1} C x_{\infty}(t)+\left(I-D_{0}\right)^{-1} D u_{\infty}(t), \\
x_{\infty}(0)= & d_{\infty},
\end{aligned}
$$

where (again a strong limit) $d_{\infty}:=\lim _{k \rightarrow \infty} d_{k}$ and the matrix $I-D_{0}$ is invertible since $r\left(D_{0}\right)<1$ by asymptotic stability in the passto-pass direction. In physical terms, this result states that under asymptotic stability in the pass-to-pass direction the dynamics can, after a sufficiently large number of passes have elapsed, be replaced by those of a $1 \mathrm{D}$ differential linear system.

Asymptotic stability in the pass-to-pass direction does not guarantee that the limit profile has acceptable along-the-pass dynamics since it can be unstable in the $1 \mathrm{D}$ linear systems sense. $A$ simple example here is $A=-1, B=1, B_{0}=1+\beta, C=1, D=$ $D_{0}=0$, where $\beta$ is a real scalar. If $\beta>0$ the limit profile for this example is unstable.

To prevent cases such as the above example from arising, one route is to demand the BIBO property for any possible value of the pass length, where mathematically this can be analyzed by letting $\alpha \rightarrow \infty$. This is the stability along-the-pass property that requires the existence of finite real scalars $M_{\infty}>0$ and $\lambda_{\infty} \in(0,1)$, which are independent of $\alpha$, such that $\left\|L_{\alpha}^{k}\right\| \leq M_{\infty} \lambda_{\infty}^{k}, k \geq$ 0 . For the processes considered here this requires [1] that (i) $r\left(D_{0}\right)<1$ (asymptotic stability in the pass-to-pass direction), (ii) all eigenvalues of $A$ have strictly negative real parts, and (iii) all eigenvalues of the transfer-function matrix $G(s)=C(s I-$ $A)^{-1} B_{0}+D_{0}$ must lie inside the unit circle in the complex plane for all $s=\imath \omega, \omega \geq 0$. In the case of the example above it is this last condition which fails when $\beta>0$. 
Each of these conditions for stability along-the-pass of an example described by (1) has a well defined interpretation, where for (i) assume that $x_{k+1}(0)=0, k \geq 0$, and then at $t=0 y_{k}(0)=$ $D_{0}^{k} y_{0}(0)$. This condition therefore requires that the initial pass profile vector sequence $\left\{y_{k}(0)\right\}_{k \geq 0}$ does not become unbounded. Condition (ii) is to be expected if the along-the-pass dynamics is to be bounded. In the case of (iii), the transfer-function involved describes the contribution of the previous pass profile to the current one [1] and in the single-input single-output (SISO) case reduces to $|G(\imath \omega)|<1$ for all $\omega \geq 0$. Hence this condition is equivalent to requiring that each frequency component of the initial pass profile is attenuated from pass-to-pass.

Stability along-the-pass demands that the signals involved are uniformly bounded as both $k \rightarrow \infty$ and $t \rightarrow \infty$, where, as discussed in more detail at the end of this section, condition (iii) for processes described by (1), is a very strict condition. Strong practical stability relaxes this by removing the uniform boundedness requirement as both $k \rightarrow \infty$ and $\alpha \rightarrow \infty$ but still demands it when (i) both $k$ and $\alpha$ are finite, (ii) the pass index $k \rightarrow \infty$ and the pass length $\alpha$ finite, and (iii) the pass index $k$ is finite and the pass length $\alpha \rightarrow \infty$. Also cases (ii) and (iii) here have practical relevance as discussed next in terms of a robotic system.

Many industrial applications use a gantry robot whose task is to collect an object from a location and place it, under synchronization, on a moving conveyor belt after a finite time has elapsed, then return to the original location to pick up the next one and so on. This is an obvious application for iterative learning control, and hence repetitive process theory, where the time taken to return to the original location can be used to update the control law using previous pass information to sequentially improve performance from pass-to-pass. Case (ii) above, that is, both $k$ and $\alpha$ are finite, is a mathematical formulation of the desire to execute this operation a very large number of times without the need to stop and hence lose throughput. Case (iii) above, that is, $k$ is finite and the pass length $\alpha \rightarrow \infty$, is the mathematical formulation where the process completes a finite number of passes but the pass length is very long and there is a requirement to control the alongthe-pass dynamics. Next these two cases are analyzed.

Under asymptotic stability in the pass-to-pass direction, the limit profile (8) as $k \rightarrow \infty$ results and is stable in the 1D linear systems senses when the eigenvalues of $A+B_{0}\left(I-D_{0}\right)^{-1} C$ have strictly negative real parts. These are necessary conditions for strong practical stability.

For any finite $k$, it is required, from consideration of the case when there is no previous pass profile contribution, that all eigenvalues of the matrix $A$ have strictly negative real parts and hence it is nonsingular. Also as $t \rightarrow \infty$

$y_{k+1}(\infty)=\left(-C A^{-1} B_{0}+D_{0}\right) y_{k}(\infty)$,

and hence $r\left(-C A^{-1} B_{0}+D_{0}\right)<1$ is required.

In summary, strong practical stability requires the following conditions to hold

[a] $r\left(D_{0}\right)<1$,

[b] all eigenvalues of the matrix $A$ have strictly negative real parts,

[c] all eigenvalues of the matrix $A+B_{0}\left(I-D_{0}\right)^{-1} C$ have strictly negative real parts, and

[d] $r\left(-C A^{-1} B_{0}+D_{0}\right)<1$.

Remark 1. To explain the term strong practical stability, first note that for 2D discrete linear systems practical stability [6] was introduced due to a recognition that the BIBO stability theory for such systems was too strong for some applications, and practical stability replaced this by the requirement the response in each direction of information propagation is stable. Practical stability can also be defined for differential linear repetitive processes described by (1) and would require conditions [a] and [b] above to hold which, as the simple example shows, is too weak in some cases.
To explain the basic difference between asymptotic stability in the pass-to-pass direction, strong practical stability and stability along-the-pass, note that $G(0)=-C A^{-1} B_{0}+D_{0}$ and also $\lim _{|s| \rightarrow \infty}$ $G(s)=D_{0}$. Consider also the case when there is zero control input, the state initial vector on each pass is zero, and, for simplicity, the SISO case. Then (as discussed prior to (9)) stability along-the-pass requires that each frequency component of the initial pass profile is attenuated from pass-to-pass, asymptotic stability in the passto-pass direction only requires this at high frequencies, and strong practical stability at both high and low frequencies together with conditions $[\mathrm{b}]$ and $[\mathrm{c}]$ above.

It is also possible to characterize strong practical stability in terms of the poles of the example considered where it is first important to note that the concept of a pole for an $n D$ linear system is much more complex than in the 1D case [7]. For the differential linear repetitive processes of the form considered here, however, the situation is less complex and can be used to explain the differences between strong practical stability and stability along-the-pass. The starting point is the characteristic polynomial for these processes defined as

$\rho(s, z)=\operatorname{det}\left(\left[\begin{array}{cc}s I-A & -B_{0} \\ -C & z I-D_{0}\end{array}\right]\right)$,

and the poles are the component-wise non-zero points in 2D complex space where the matrix whose determinant appears on the right-hand side fails to have full rank, that is, they are given by

$\rho(s, z)=0$,

and the set $\left\{a_{1}, a_{2}\right\}$ that satisfy this last equation is termed the pole variety. Also stability along-the-pass holds if, and only if,

$\rho(s, z) \neq 0, \quad \operatorname{Re}(s) \geq 0, \quad|z| \geq 1$.

The poles are given by the vanishing of a single 2D non-unit polynomial and the pole variety is guaranteed to be a 1D geometric set in 2D complex space. Note also that the pole variety must be complex, even though the entries of the matrices $A, B_{0}, C$ and $D_{0}$ are real. This is essential in order to capture the full exponentialtype dynamics of the process.

The poles of these processes can be interpreted in terms of exponential trajectories, which in the case considered here have a clear physical interpretation, and stability along-the-pass requires no poles with $\operatorname{Re} s \geq 0$ and $|z| \geq 1$, which is a direct generalization of the 1D linear systems case. For strong practical stability, it is easy to see that condition [c] is equivalent to $\rho(s, 1) \neq 0, \operatorname{Re} s \geq 0$, and condition [d] to $\rho(0, z) \neq 0,|z| \geq 1$. Hence for this stability property the only exponential trajectories considered are identical to those for 1D discrete and differential linear systems respectively, and these are clearly a subset of those given by (11).

In terms of design to track a given reference vector, imposing the requirement for stability along-the-pass requires the control law to achieve the required level of attenuation over the complete frequency range and this, by comparison with the 1D linear systems case, is most likely to result in a very difficult design problem. In such cases, strong practical stability may lead to acceptable design, especially for applications where an unstable limit profile is not acceptable and/or some control is required over the along-the-pass dynamics.

\section{LMI based stability tests}

The conditions for strong practical stability can, assuming no numerical problems with computing the eigenvalues of the matrices involved, be easily checked for a given example. Suppose, however, that the task is to ensure this property by application of a control law, see also the next section, of the form

$u_{k+1}(t)=K_{1} x_{k+1}(t)+K_{2} y_{k}(t)$ 
which is a combination of current pass state feedback plus a feedforward term from the previous pass profile, in keeping with the fact that the use of only current pass state or pass profile vector activated control laws cannot stabilize the process dynamics in all but a few restrictive special cases. On applying this control law to (1), the process state-space model matrices $A, B_{0}, C, D_{0}$ are mapped to $A+B K_{1}, B_{0}+B K_{2}, C+D K_{1}$, and $D_{0}+D K_{2}$, respectively. Hence design to satisfy conditions [a] and [b] for the controlled process is simply the $1 \mathrm{D}$ pole placement problem for discrete and differential linear systems respectively. The case for conditions [c] and $[\mathrm{d}]$ is far from clear and hence as a preliminary step to overall control law design novel use is made of results from 1D descriptor differential and discrete linear systems theory for the state-space models

$$
\begin{aligned}
& E \dot{x}(t)=\widehat{A} x(t)+\widehat{B} u(t), \\
& y(t)=\widehat{C} x(t)+\widehat{D} u(t),
\end{aligned}
$$

and

$E x(h+1)=\widehat{A} x(h)+\widehat{B} u(h)$,

$y(h)=\widehat{C} x(h)+\widehat{D} u(h)$,

respectively, where $E$ is a square matrix that could be singular. This will lead to stability tests in terms of LMIs which (see the next section) lead to control law design algorithms.

Condition $[\mathrm{c}]$ is equivalent to the stability of the 1D descriptor singular differential linear system with state-space model

$E_{1} \dot{z}(t)=A_{1} z(t)+\Pi u(t)$,

where

$A_{1}=\left[\begin{array}{cc}A & B_{0} \\ C & D_{0}-I\end{array}\right], \quad E_{1}=\left[\begin{array}{ll}I & 0 \\ 0 & 0\end{array}\right]$,

$\Pi=\left[\begin{array}{l}B \\ D\end{array}\right], \quad z(t)=\left[\begin{array}{l}x_{\infty}(t) \\ y_{\infty}(t)\end{array}\right]$

which follows immediately on applying appropriate elementary operations to the characteristic matrix $s I-\left(A+B_{0}\left(I-D_{0}\right)^{-1} C\right)$. Also (9) can be rewritten as

$0=A x_{k+1}(\infty)+B_{0} y_{k}(\infty)$,

$y_{k+1}(\infty)=C x_{k+1}(\infty)+D_{0} y_{k}(\infty)$.

Hence condition [d] is equivalent to the stability requirement for the following 1D descriptor discrete linear system

$E_{2} z(h+1)=A_{2} z(h)+\Pi u(h)$,

where

$A_{2}=\left[\begin{array}{cc}0 & B_{0} \\ 0 & D_{0}\end{array}\right], \quad E_{2}=\left[\begin{array}{ll}-A & 0 \\ -C & I\end{array}\right]$,

$z(h)=\left[\begin{array}{l}x_{h}(\infty) \\ y_{h}(\infty)\end{array}\right]$

and the descriptor matrix $E_{2}$ is nonsingular when condition [b] holds. The following definitions are also required.

Definition 1. A 1D differential descriptor linear system of the form (14) is termed regular [8] if the state-space equation (14) has one single solution, that is, if $\operatorname{det}(s E-\widehat{A})$ is not identically zero.

Definition 2. A 1D regular differential descriptor linear system (14) is termed admissible [8] if it is asymptotically stable (the finite roots of $\operatorname{det}(s E-\widehat{A})$ lie inside the open left-half complex plane) and impulse-free (the possible infinite roots do not generate impulses in the response, that is $\operatorname{deg}(\operatorname{det}(s E-\widehat{A}))=\operatorname{rank} E)$.

Either asymptotic stability or impulse freeness implies regularity and hence admissibility implies regularity. Also if $E$ is nonsingular the $1 D$ linear descriptor system $E z(p+1)=\hat{A} z(p)$ can be easily rewritten in the conventional state-space form as $z(p+1)=$ $E^{-1} \hat{A} z(p)$ and the following result is useful in obtaining necessary and sufficient conditions for strong practical stability.

Lemma 1 ([9,10]). Consider a $1 D$ linear descriptor system $E z(p+$ $1)=\hat{A} z(p)$ with $E$ nonsingular. Then the system $z(p+1)=E^{-1} \hat{A} z(p)$ is stable if, and only if there exists a matrix $\hat{Q}>0$ and a nonsingular matrix $\hat{G}$ such that

$\left[\begin{array}{cc}-\hat{Q} & \hat{A}^{T} E^{-T} \hat{G}^{T} \\ \hat{G} E^{-1} \hat{A} & \hat{Q}-\hat{G}-\hat{G}^{T}\end{array}\right]<0 \Leftrightarrow-\hat{Q}+\hat{A}^{T} E^{-T} \hat{Q} E^{-1} \hat{A}<0$.

The right-hand side LMI in the above result is due to [9] and the equivalence with the one on the left-hand side is proved in [10]. Also admissibility can be determined by the following alternative to (16) and (17), see, for example, [11].

Lemma 2. The system described by (16) and (17) is admissible if, and only if, there exists a nonsingular matrix $G$ such that

$E_{1}^{T} G=G^{T} E_{1} \geq 0$,

$G^{T} A_{1}+A_{1}^{T} G<0$.

The following result gives computable necessary and sufficient conditions for strong practical stability.

Theorem 1. A differential linear repetitive process described by (1) is strongly practically stable if, and only if, there exist matrices $W_{1}>0$, $W_{2}>0, Q_{2}>0$, and a nonsingular matrix

$G=\left[\begin{array}{cc}G_{11} & 0 \\ G_{21} & G_{22}\end{array}\right]$,

with $G_{11}>0$ such that the following LMIs are feasible

$\left[\begin{array}{cc}-W_{1} & W_{1}^{T} D_{0}^{T} \\ D_{0} W_{1} & -W_{1}\end{array}\right]<0$

$A^{T} W_{2}+W_{2} A<0$,

$G^{T} A_{1}+A_{1}^{T} G<0$,

$-Q_{2}+A_{2}^{T} E_{2}^{-T} Q_{2} E_{2}^{-1} A_{2}<0$.

Proof. The LMIs (23) and (24) are well known conditions (see, for example, [12,13]) for the stability of 1D discrete and differential linear systems and correspond to [a] and [b] for strong practical stability.

The equivalence between the LMI (25) and condition [c] follows immediately on applying Lemma 2 to (16) and (17). In particular, (22) requires that $G$ is nonsingular with $G_{11}>0$ and $G_{12}=0$. Therefore (16) is admissible and hence stable. Finally, the equivalence of the LMI (26) and condition [d] follows immediately on applying the right-hand side LMI of Lemma 1 to (19) and (20) with $\hat{Q}=Q_{2}$, and $E=E_{2}$, where condition [b] ensures that $E_{2}$ is nonsingular.

\section{Control law design}

Applying the control law (13) to (1) gives the controlled process state-space model

$\dot{x}_{k+1}(t)=\left(A+B K_{1}\right) x_{k+1}(t)+\left(B_{0}+B K_{2}\right) y_{k}(t)$,

$y_{k+1}(t)=\left(C+D K_{1}\right) x_{k+1}(t)+\left(D_{0}+D K_{2}\right) y_{k}(t)$,

and the following result gives the necessary and sufficient conditions for strong practical stability.

Theorem 2. A differential linear repetitive process described by (27) is strongly practically stable if, and only if,

[e] $r\left(D_{0}+D K_{2}\right)<1$, 
[f] all eigenvalues of $A+B K_{1}$ have strictly negative real parts,

[g] all eigenvalues of $A+B K_{1}+\left(B_{0}+B K_{2}\right)\left(I-D_{0}-D K_{2}\right)^{-1}\left(C+D K_{1}\right)$ have strictly negative real parts, and

[h] $r\left(D_{0}+D K_{2}-\left(C+D K_{1}\right)\left(A+B K_{1}\right)^{-1}\left(B_{0}+B K_{2}\right)\right)<1$.

Control law design based on Theorem 2 is somewhat complex since only the two control law matrices $K_{1}$ and $K_{2}$ are available for design to simultaneously satisfy the four conditions [e]-[h]. This difficulty is removed by the following result.

Theorem 3. Suppose that a control law of the form (13) is applied to a differential linear repetitive process described by (1). Then the following hold.

(a) The controlled process with state-space model (13) is strongly practically stable if there exist matrices $Q_{1}>0, Q_{2}>0, S_{1}>0, a$ nonsingular matrix $S_{2}$, and rectangular matrices $\widetilde{N}_{1}=\left[\begin{array}{ll}N_{1} & 0\end{array}\right], \widetilde{N}_{2}=$ $\left[\begin{array}{ll}0 & N_{2}\end{array}\right]$ and $N=\left[\begin{array}{ll}N_{1} & N_{2}\end{array}\right]$ such that the following LMIs are feasible

$S^{T} A_{1}^{T}+N^{T} \Pi^{T}+A_{1} S+\Pi N<0$,

$\left[\begin{array}{cc}-Q_{2} & S^{T} A_{2}^{T}+\widetilde{N}_{2}^{T} \Pi^{T} \\ A_{2} S+\Pi \tilde{N}_{2} & Q_{2}-\left(E_{2} S-\Pi \tilde{N}_{1}\right)-\left(E_{2} S-\Pi \tilde{N}_{1}\right)^{T}\end{array}\right]<0$,

where $S=\left[\begin{array}{cc}S_{1} & 0 \\ 0 & S_{2}\end{array}\right]$ and $\Pi$ is defined in (17).

(b) Suppose that the LMIs of (28) and (29) hold. Then stabilizing control law matrices can be computed using

$K_{1}=N_{1} S_{1}^{-1}, \quad K_{2}=N_{2} S_{2}^{-1}$.

Proof. First it is shown that (28) and (29) respectively guarantee that $[\mathrm{g}]$ and $[\mathrm{h}]$ of Theorem 2 hold.

Condition [ $\mathrm{g}]$ is equivalent to the stability of the closed-loop 1D singular differential linear system with state-space model

$E_{1} \dot{z}(t)=\left(A_{1}+\Pi\left[\begin{array}{ll}K_{1} & K_{2}\end{array}\right]\right) z(t)$.

The result now follows on applying the dual form of (25) in Theorem 1 to this last state-space model.

Condition $[\mathrm{h}]$ is equivalent to the stability of the $1 \mathrm{D}$ descriptor discrete linear system (19) with the control law

$u(h)=\left[\begin{array}{ll}K_{1} & 0\end{array}\right] z(h+1)+\left[\begin{array}{ll}0 & K_{2}\end{array}\right] z(h)$,

applied. Given (30), introduce the additional matrix variables $N_{1}=$ $K_{1} S_{1}$ and $N_{2}=K_{2} S_{2}$ into the LMI (29) to obtain

$\left[\begin{array}{cc}-Q_{2} & S_{2}^{T} A_{2 \text { new }}^{T} \\ A_{2 \text { new }} S_{2} & Q_{2}-E_{2 \text { new }} S_{2}-S_{2}^{T} E_{2 \text { new }}^{T}\end{array}\right]<0$,

with $A_{2 \text { new }}=A_{2}+\Pi\left[\begin{array}{ll}0 & K_{2}\end{array}\right]$ and $E_{2 n e w}=E_{2}-\Pi\left[\begin{array}{cc}K_{1} & 0\end{array}\right]$. By congruence, this is equivalent to

$\left[\begin{array}{cc}-Q_{2} & S_{2}^{T} A_{2 \text { new }}^{T} E_{2 \text { new }}^{-T} \\ E_{2 \text { new }}^{-1} A_{2 \text { new }} S_{2} & Q_{2}-S_{2}-S_{2}^{T}\end{array}\right]<0$,

which is the dual form of the first LMI in (21). Hence, on making use of the result in Lemma 1 ,

$-Q_{2}+E_{2 \text { new }}^{-1} A_{2 \text { new }} Q_{2} A_{2 \text { new }}^{T} E_{2 \text { new }}^{-T}<0$,

and this is the dual version of (26) of Theorem 1 when applied with $A_{2 \text { new }}$ and $E_{2 \text { new }}$. Hence condition [h] holds by Theorem 1 applied to (27).

Next, it is required to show that the LMIs (28) and (29) guarantee that [e] and [f] hold, where, using (30) and Theorem 1 applied to (27), these are equivalent to

$\left[\begin{array}{cc}-W_{1} & S_{2}^{T} D_{0}^{T}+N_{2}^{T} D^{T} \\ D_{0} S_{2}+D N_{2} & W_{1}-S_{2}-S_{2}^{T}\end{array}\right]<0$, and

$S_{1}^{T} A^{T}+N_{1}^{T} B^{T}+A S_{1}+B N_{1}<0$,

respectively, where the second is stated in its dual form.

The LMI of (29) can be written as

$$
\begin{aligned}
& {\left[\begin{array}{cccc}
-Q_{211} & -Q_{212} & 0 & 0 \\
-Q_{212}^{T} & -Q_{222} & S_{2}^{T} B_{0}^{T}+N_{2}^{T} B^{T} & S_{2}^{T} D_{0}^{T}+N_{2}^{T} D^{T} \\
0 & B_{0} S_{2}+B N_{2} & \Upsilon & Q_{212}+S_{1}^{T} C^{T}+N_{1}^{T} D^{T} \\
0 & D_{0} S_{2}+D N_{2} & Q_{212}^{T}+C S_{1}+D N_{1} & Q_{222}-S_{2}-S_{2}^{T}
\end{array}\right]} \\
& <0 \text {, }
\end{aligned}
$$

where

$\Upsilon=Q_{211}-S_{1}-S_{1}^{T}+A S_{1}+B N_{1}+S_{1}^{T} A^{T}+B^{T} N_{1}^{T}$.

Since a real symmetric matrix is negative definite if, and only if, all of its principal minors are negative definite, (38) can only hold when

$$
\left[\begin{array}{ccc}
-Q_{222} & S_{2}^{T} B_{0}^{T}+N_{2}^{T} B^{T} & S_{2}^{T} D_{0}^{T}+N_{2}^{T} D^{T} \\
B_{0} S_{2}+B N_{2} & \Upsilon & Q_{212}+S_{1}^{T} C^{T}+N_{1}^{T} D^{T} \\
D_{0} S_{2}+D N_{2} & Q_{212}^{T}+C S_{1}+D N_{1} & Q_{222}-S_{2}-S_{2}^{T}
\end{array}\right]<0 .
$$

Equivalently, by obvious row and column permutations in (39), it is required that

$$
\left[\begin{array}{ccc}
Q_{222}-S_{2}-S_{2}^{T} & D_{0} S_{2}+D N_{2} & Q_{212}^{T}+C S_{1}+D N_{1} \\
\left(D_{0} S_{2}+D N_{2}\right)^{T} & -Q_{222} & S_{2}^{T} B_{0}^{T}+N_{2}^{T} B^{T} \\
\left(Q_{212}^{T}+C S_{1}+D N_{1}\right)^{T} & B_{0} S_{2}+D N_{2} & \Upsilon
\end{array}\right]<0 .
$$

For (40) to hold it is necessary that

$\left[\begin{array}{cc}Q_{222}-S_{2}-S_{2}^{T} & D_{0} S_{2}+D N_{2} \\ \left(D_{0} S_{2}+D N_{2}\right)^{T} & -Q_{222}\end{array}\right]<0$.

Left- and right-multiplying this last inequality by $\left[\begin{array}{ll}0 & I \\ I & 0\end{array}\right]$ gives (36) with $W_{2}=Q_{222}$.

Finally, rewrite the LMI (28) as

$$
\begin{aligned}
& {\left[\begin{array}{lc}
S_{1} A^{T}+N_{1}^{T} B^{T}+A S_{1}+B N_{1} & B_{0} S_{2}+B N_{2}+S_{1} C^{T}+N_{1}^{T} D^{T} \\
C S_{1}+D N_{1}+S_{2}^{T} B_{0}^{T}+N_{2}^{T} B^{T} & D_{0} S_{2}+D N_{2}+S_{2}^{T} D_{0}+N_{2}^{T} D^{T}-2 I
\end{array}\right]} \\
& \quad<0,
\end{aligned}
$$

and if this last LMI is feasible (37) holds.

This result is much simpler than that of [14] and hence more computationally tractable and less conservative. Also, the LMIs are not parameterized and there is no parameter which has to be tuned to achieve appropriate controlled process dynamics.

\section{Conclusions}

The existing stability theory for differential linear repetitive processes leads to conditions that can be expressed in terms of well known 1D discrete and differential linear systems tests. The strongest form is stability along-the-pass, which is very stringent in terms of control law design. This paper has developed strong practical stability as an alternative, where the conditions for this property are in terms of LMIs that extend to allow the design of control laws.

\section{References}

[1] E. Rogers, K. Gałkowski, D.H. Owens, Control Systems Theory and Applications for Linear Repetitive Processes, in: Lecture Notes in Control and Information Sciences, vol. 349, Springer, Berlin, Germany, 2007.

[2] D.A. Bristow, M. Tharayil, A.G. Alleyne, A survey of iterative learning control, IEEE Control Systems Magazine 26 (3) (2006) 96-114. 
[3] Hyo-Sung Ahn, YangQuan Chen, K.L. Moore, Iterative learning control: brief survey and categorization 1998-2004, IEEE Transactions on Systems, Man and Cybernetics Part C 37 (6) (2007) 1099-1121.

[4] P.D. Roberts, Numerical investigation of a stability theorem arising from 2-dimensional analysis of an iterative optimal control algorithm, Multidimensional Systems and Signal Processing 11 (1-2) (2000) 109-124.

[5] L. Hładowski, K. Gałkowski, Z. Cai, E. Rogers, C.T. Freeman, P.L. Lewin, Experimentally supported 2D systems based iterative learning control law design for error convergence and performance, Control Engineering Practice 18 (4) (2010) 339-348.

[6] P. Agathoklis, L.T. Bruton, Practical-BIBO stability of $n$-dimensional discrete systems, IEE Proceedings. Part G. Electronic Circuits and Systems 130 (6) (1983) 236-242.

[7] J. Wood, O. Oberst, E. Rogers, D.H. Owens, A behavioural approach to the pole structure of one-dimensional and multidimensional linear systems, SIAM Journal on Control and Optimization 38 (2) (2000) 627-661.
[8] L. Dai, Singular Control Systems, in: Lecture Notes in Control and Information Sciences, vol. 118, Springer, Berlin, Germany, 1989.

[9] P. Stein, Some general theorems on iterants, Journal of Research of the National Bureau of Standards 48 (1952) 82-83.

[10] M.C. de Oliveira, J. Bernussou, J.C. Geromel, A new discrete-time robust stability condition, Systems and Control Letters 39 (4) (1999) 261-265.

[11] S. Xu, J. Lam, Robust Control and Filtering of Singular Systems, in: Lecture Notes in Control and Information Sciences, vol. 332, Springer, Berlin, Germany, 2006

[12] G. Garcia, J. Bernussou, Pole assignment for uncertain systems in a specified disk by state feedback, IEEE Transactions on Automatic Control 40 (1) (1995) 184-190.

[13] M. Chilali, P. Gahinet, $H_{\infty}$ design with pole placement constraints: an LM approach, IEEE Transactions on Automatic Control 41 (3) (1996) 358-367.

[14] P. Dabkowski, K. Gałkowski, E. Rogers, Strong practical stability and stabilization of 2D differential-discrete linear systems, in: Proceedings of 47 th IEEE Conference on Decision and Control, 2008, pp. 2385-2390. 